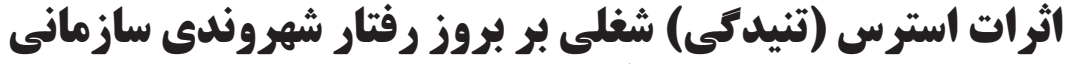

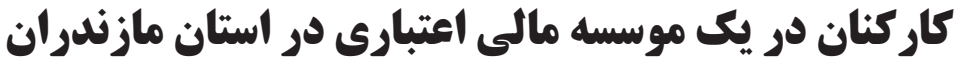

حسين صمدى ميار كلائى ا:*ّ، حمزه صمدى ميار كلائى '

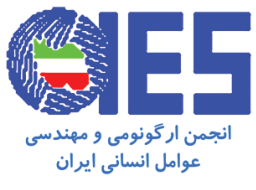

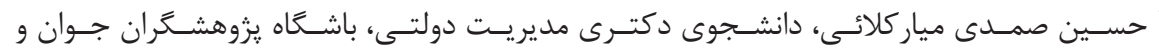

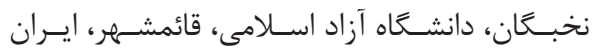

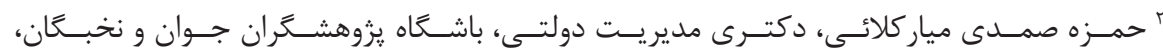

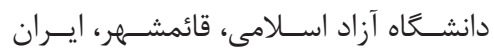

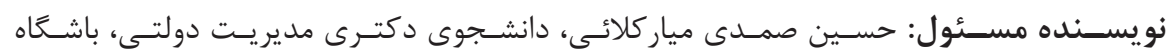

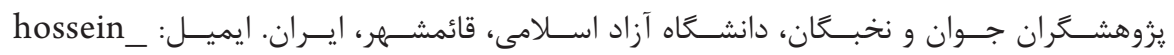

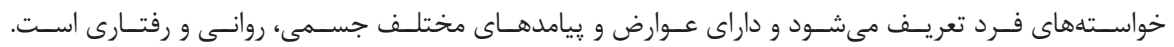

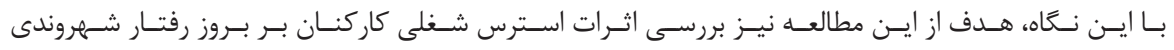

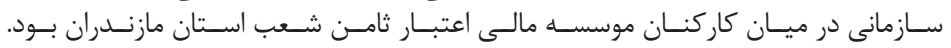

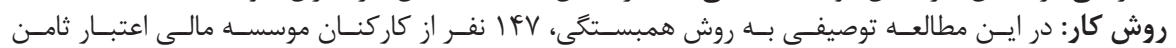

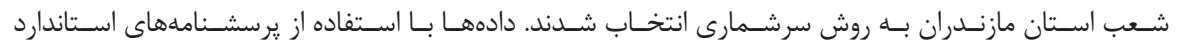

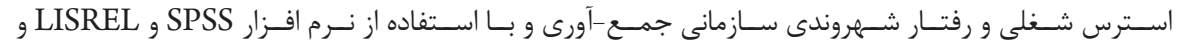

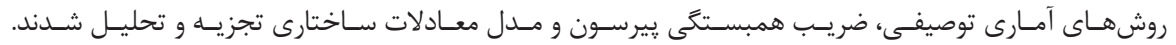

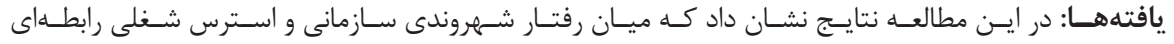

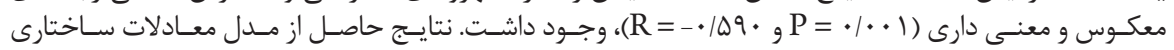

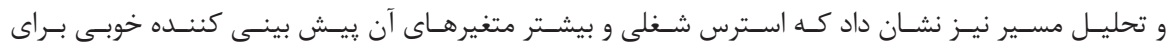

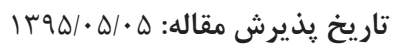

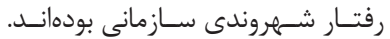

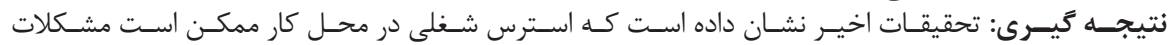

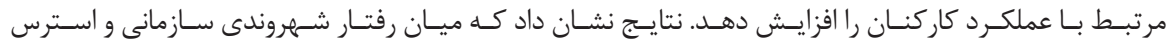

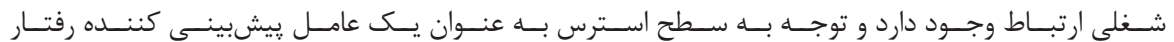

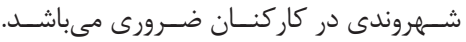

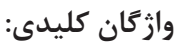

استرس شغلى رفتار شهروندى سازمانى محيط كارى كاركنان تمامى حقوق نشر براى دانشكاه علوم يزشكى

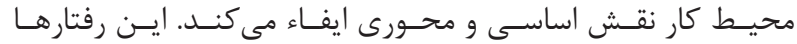

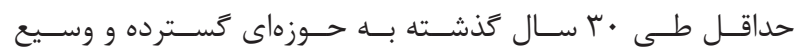

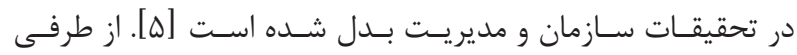

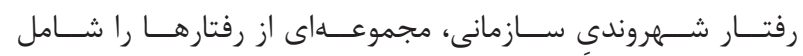

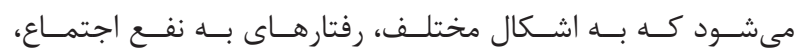

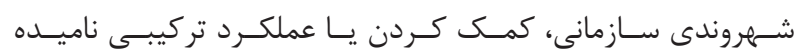

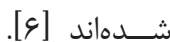

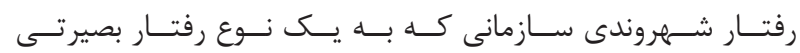

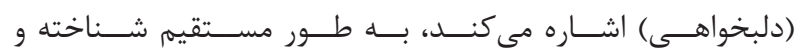

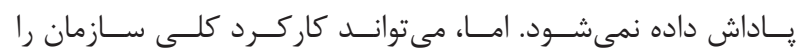

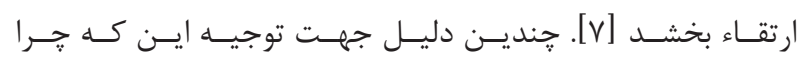

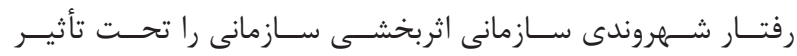

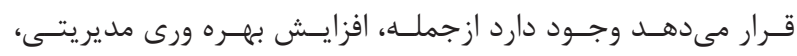

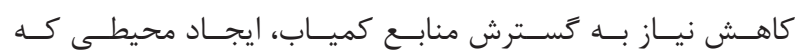

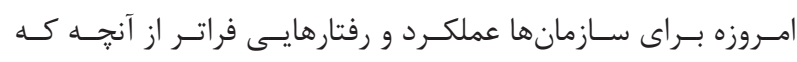

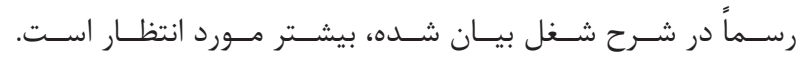

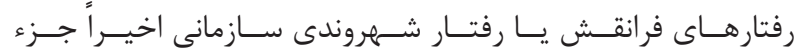

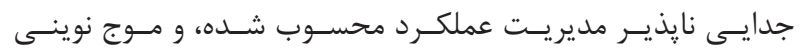

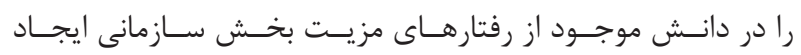

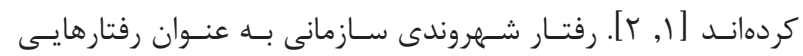

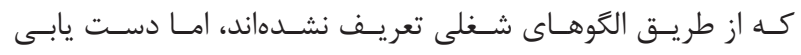

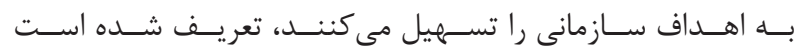

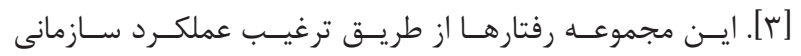

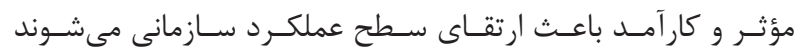

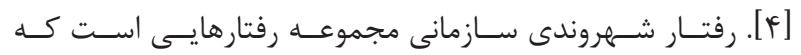

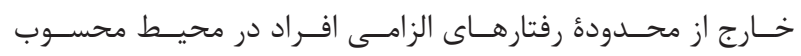

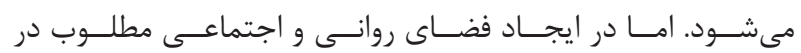




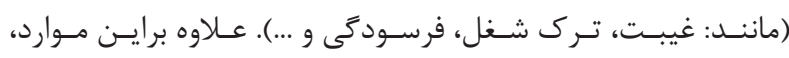

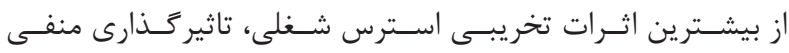

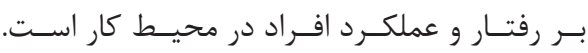

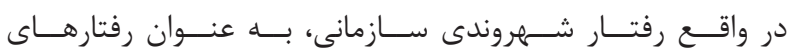

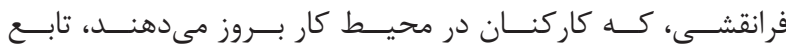

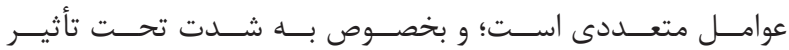

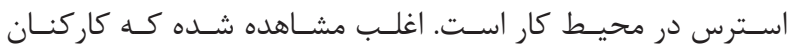

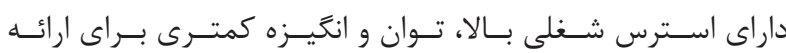

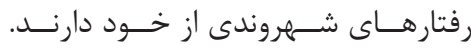

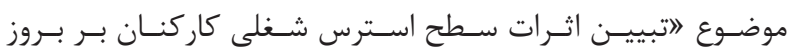

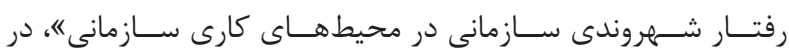

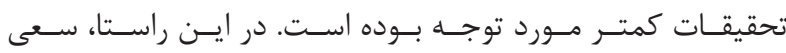

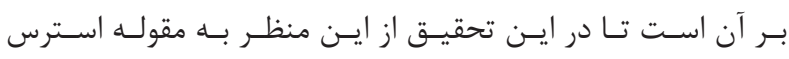

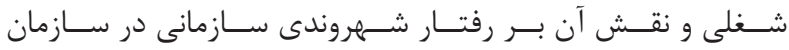

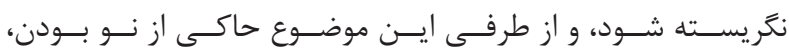

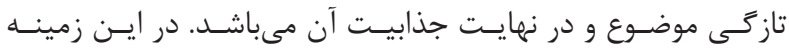

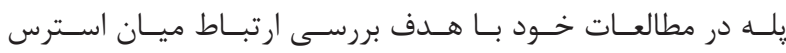

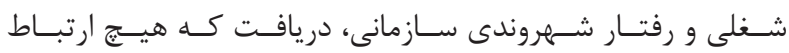

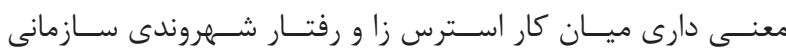

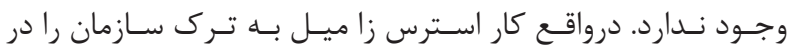

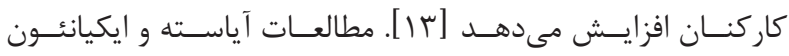

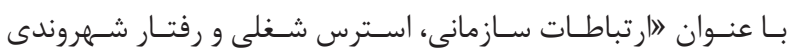

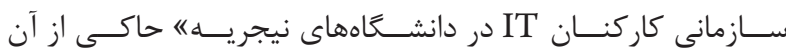

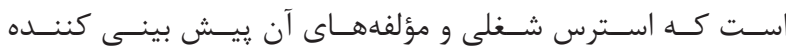

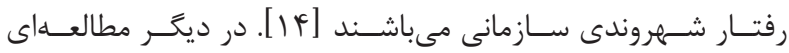

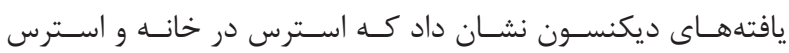

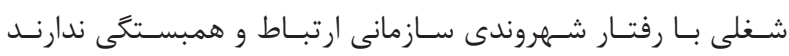

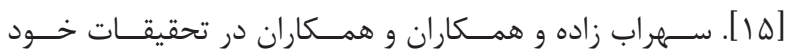

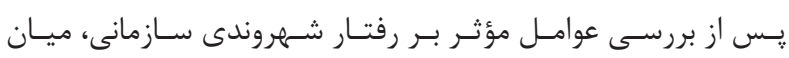

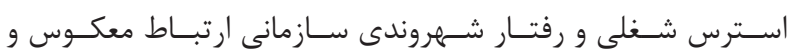

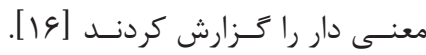

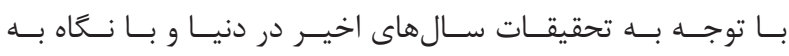

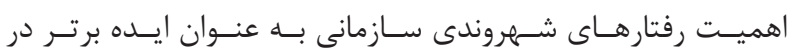

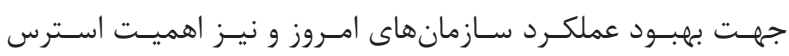

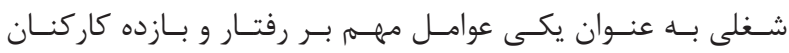

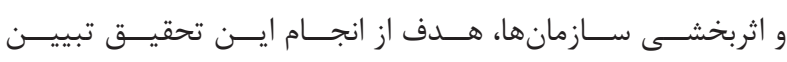

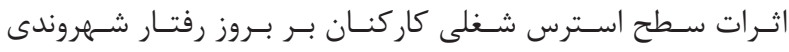

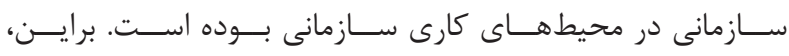

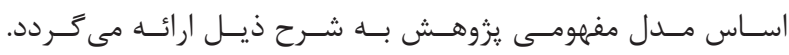

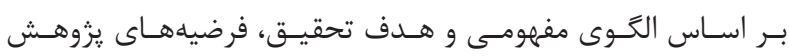

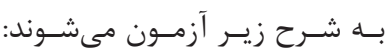

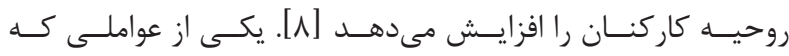

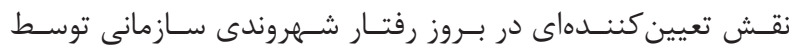

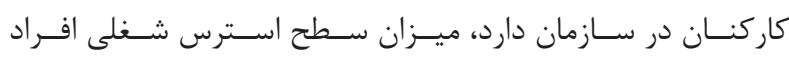

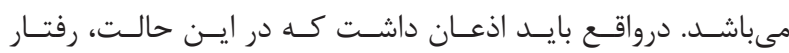

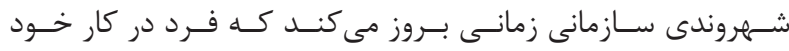

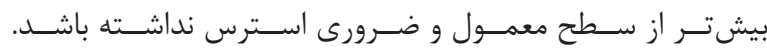

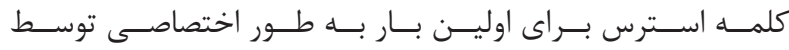

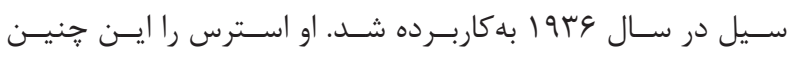

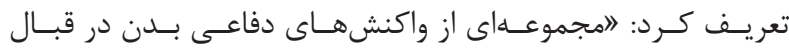

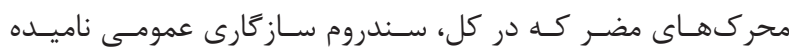

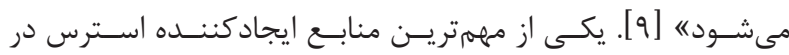

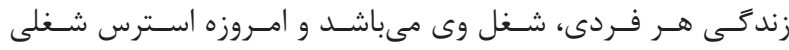

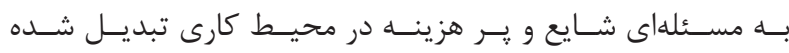

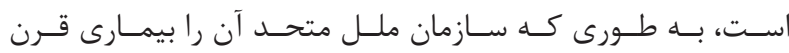

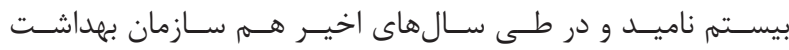

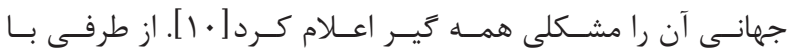

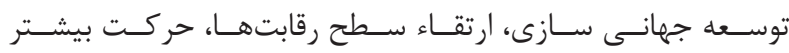

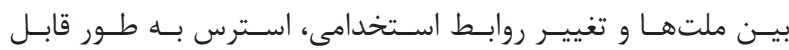

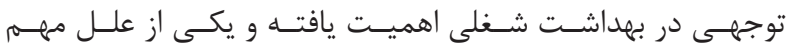

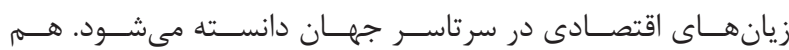

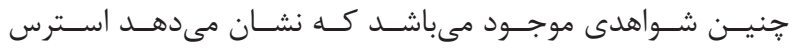

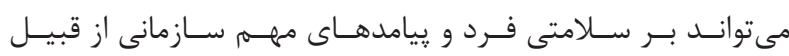

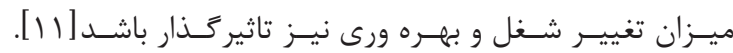

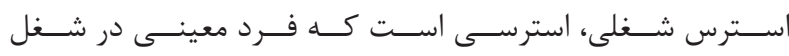

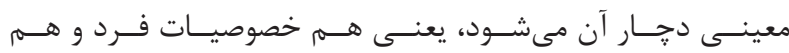

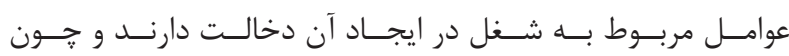

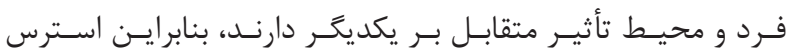

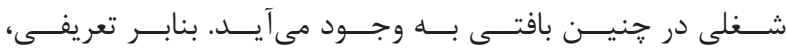

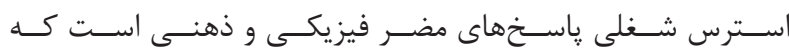

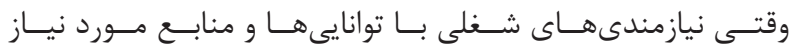

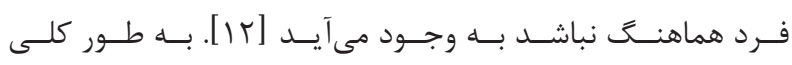

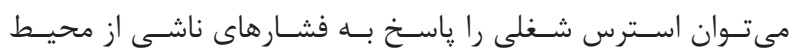

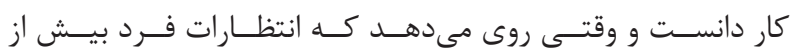

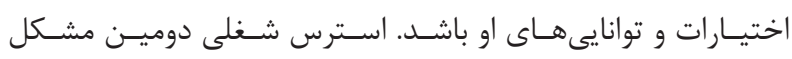

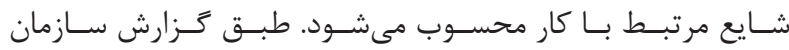

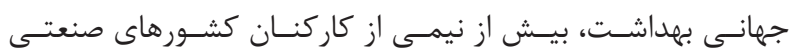

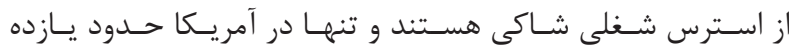

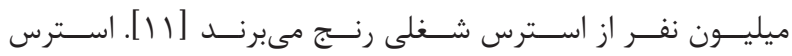

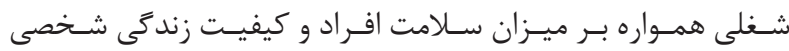

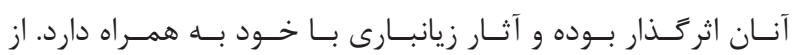

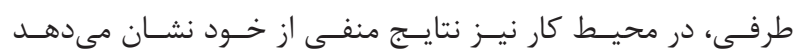




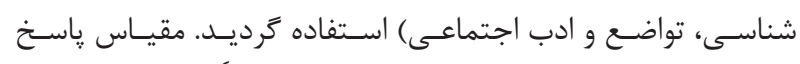

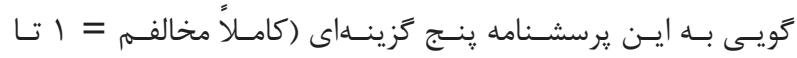

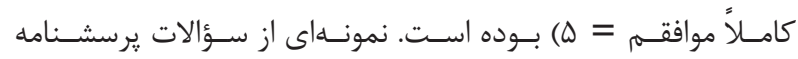

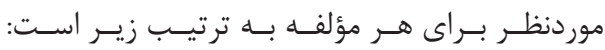

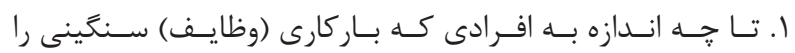

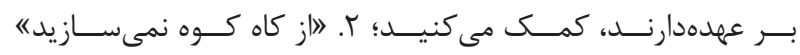

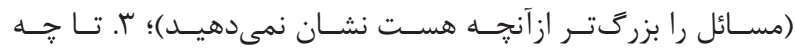

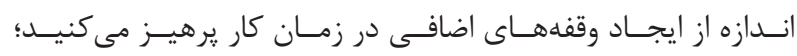

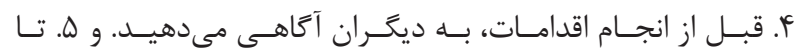

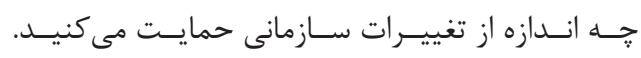

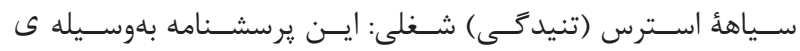

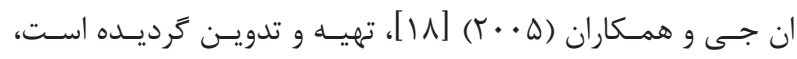

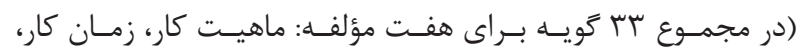

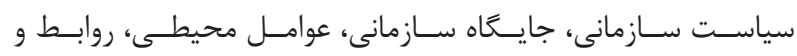

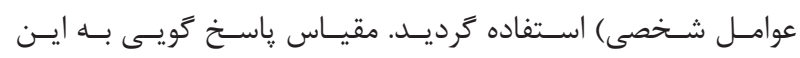

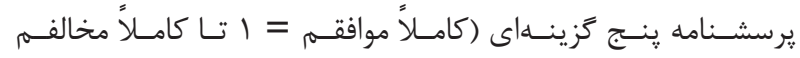

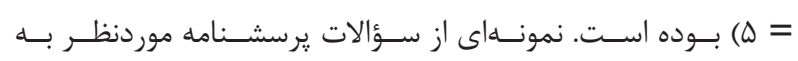
ترتيـب زيــر اسـت:

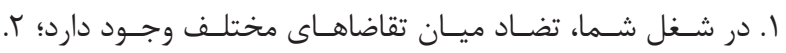

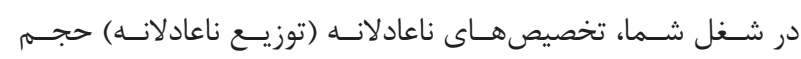

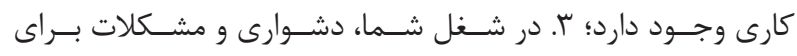

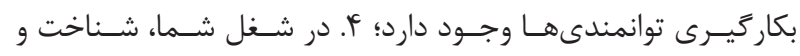

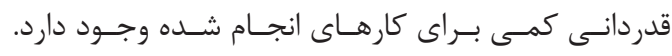

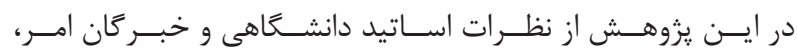

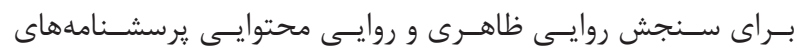

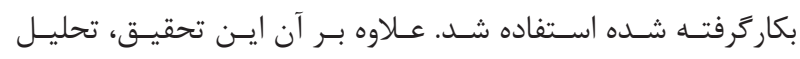

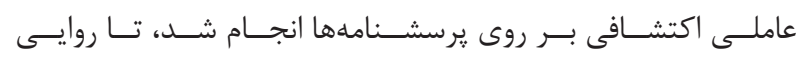

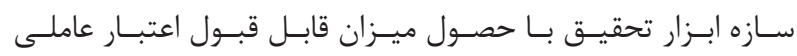

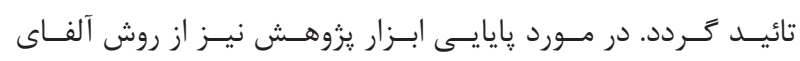

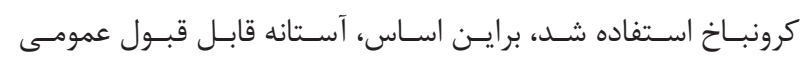

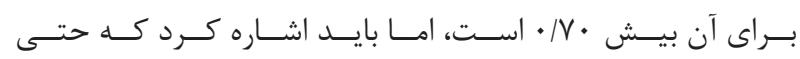

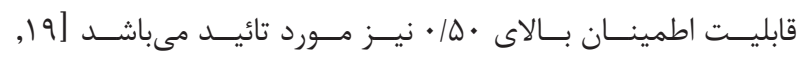

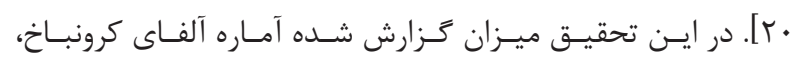

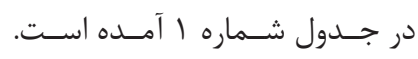

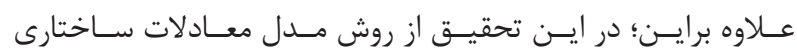

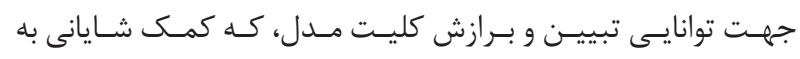

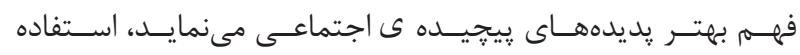

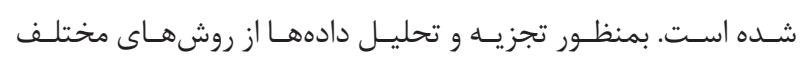

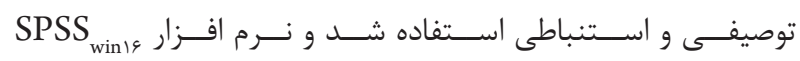

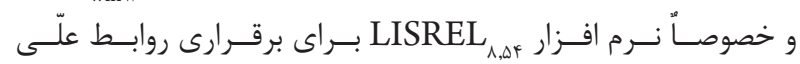

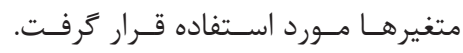

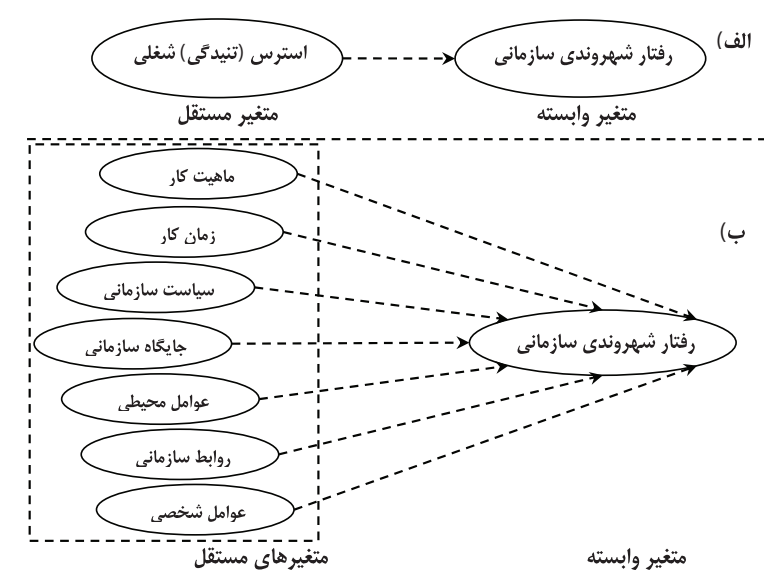

تصوير l. ترسيم خارجوب مفهومى تحقيق

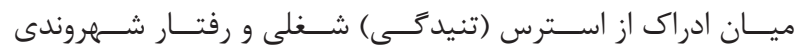

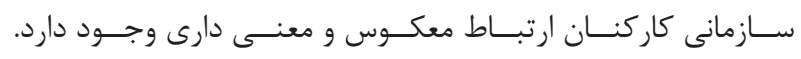

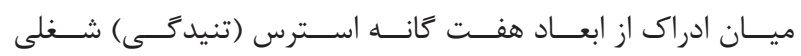

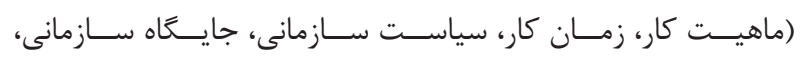

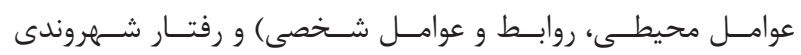

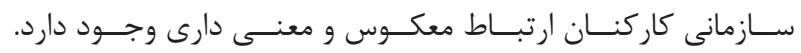

\section{روش كار}

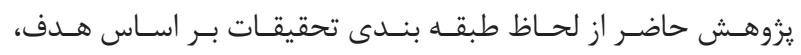

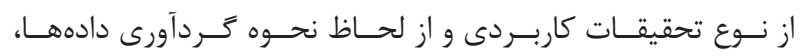

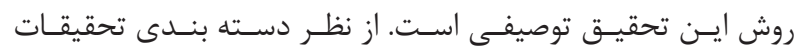

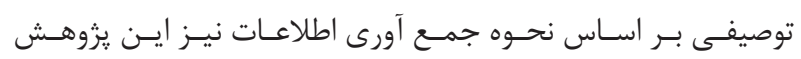

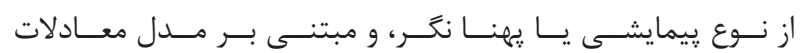

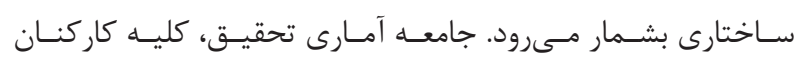

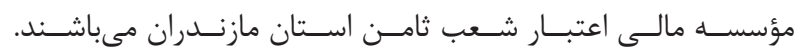

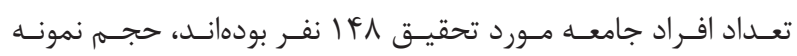

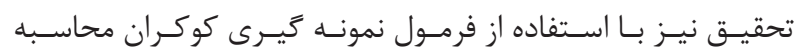

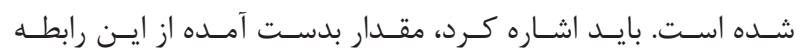

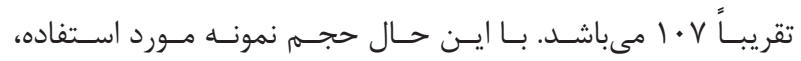

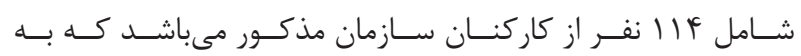

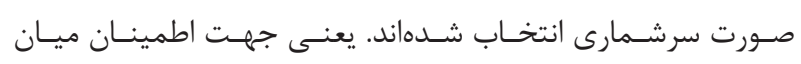

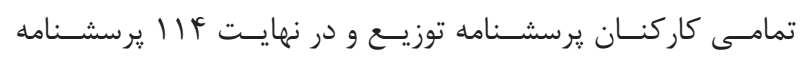

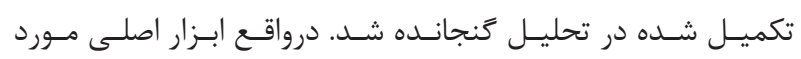

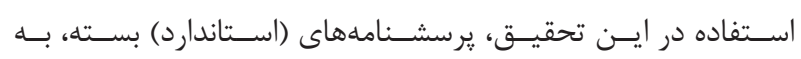
شـرح ذيـل بـوده اسـت:

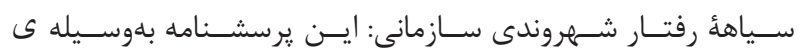

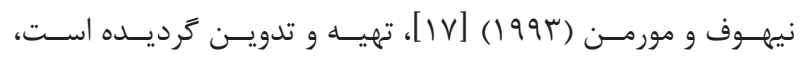

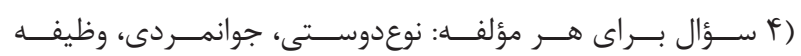




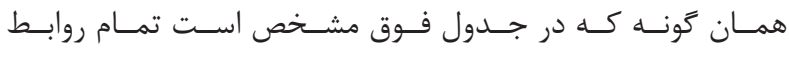

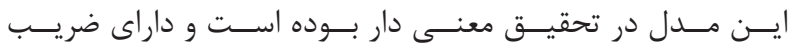

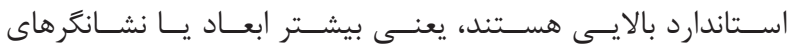

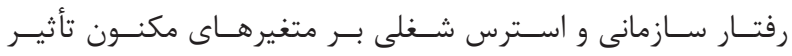

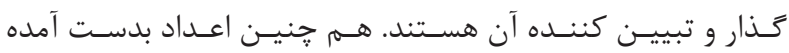

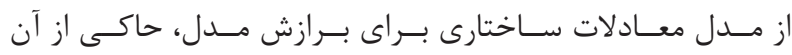

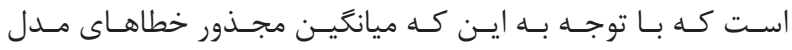

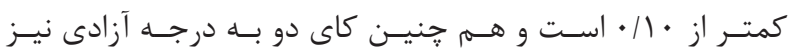

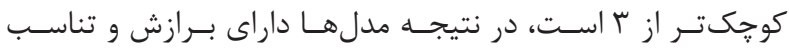

$$
\text { بالايسى مىباشـنـد. }
$$

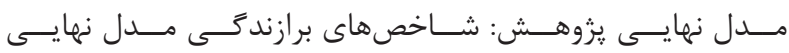

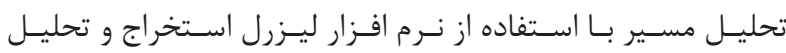

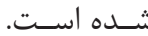
نتايسج خروجـى ليـزرل و تحليـل قســمت تخميـن اسـتاندارد و

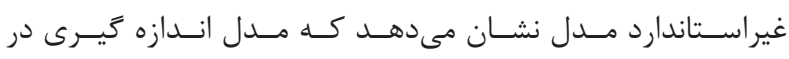

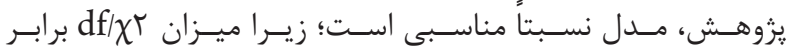

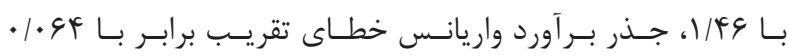

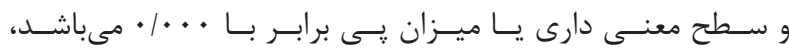

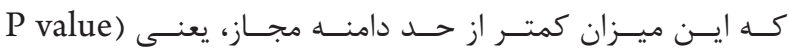

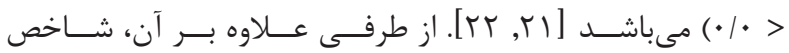

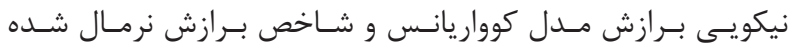

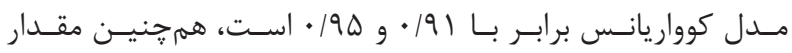

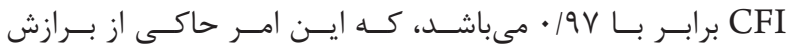

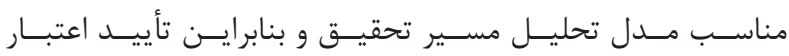

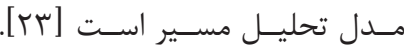

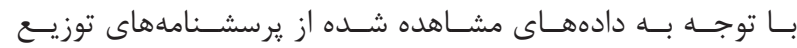

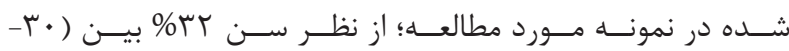

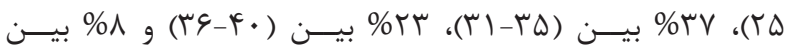
(

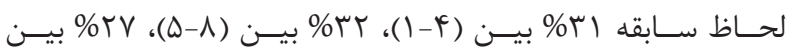

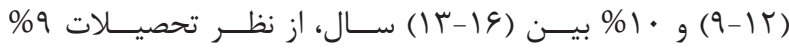

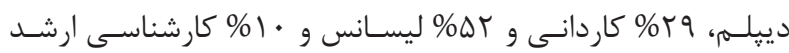

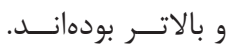

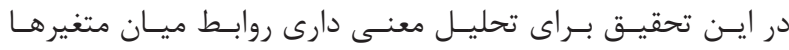

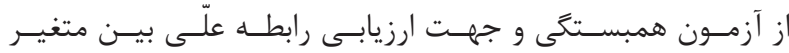

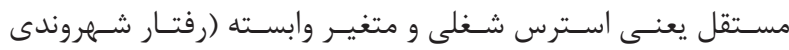

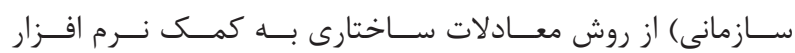

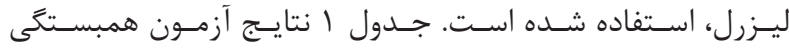

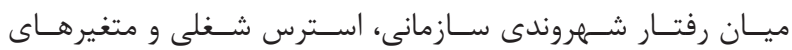

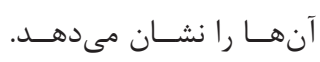

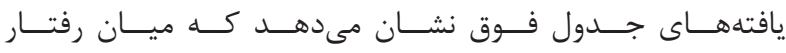

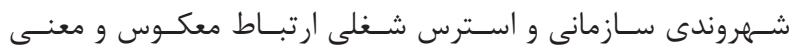

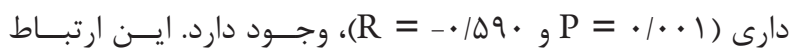

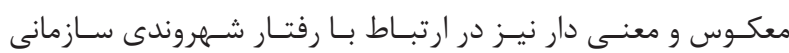

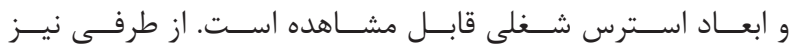

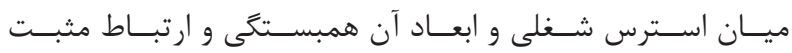

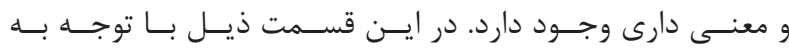

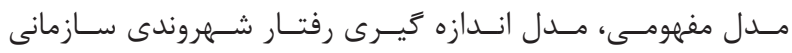

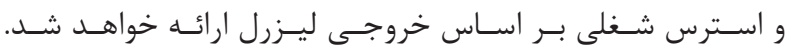

\begin{tabular}{|c|c|c|c|c|c|c|c|c|c|}
\hline \multirow[b]{2}{*}{9} & \multirow[b]{2}{*}{$\wedge$} & \multirow[b]{2}{*}{$\checkmark$} & \multirow[b]{2}{*}{9} & \multirow[b]{2}{*}{$\Delta$} & \multirow[b]{2}{*}{ F } & \multicolumn{4}{|c|}{ جدول ا: پايايى و ماتريس همبستگى براى متغيرهاى يزوهش } \\
\hline & & & & & & $r$ & r & 1 & متغير ها \\
\hline & & & & & & & & $(\cdot / 94)$ & 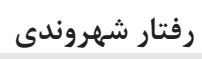 \\
\hline & & & & & & & 1 & $-\cdot|\& \Delta| \cdots$ & ماهيت كار \\
\hline & & & & & & 1 & $.199 V^{* a s}$ & $-\cdot / \Upsilon_{\Lambda} \varphi^{m+n}$ & زمان كار \\
\hline & & & & & 1 & 吾 & $\cdot / T r V^{* a s}$ & $-\cdot / 1 \wedge V^{*}$ & سياست سازمان \\
\hline & & & & 1 & $\cdot|r F|^{* n}$ & $\cdot \mid V T I^{*}$ & •/VG4" & 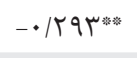 & جايعًاه سازمانى \\
\hline & & & 1 & $\cdot / V \Delta \cdot *$ & $\cdot / r \Lambda r^{* *}$ & $\cdot 19 \cdot \mu^{m}$ & $\cdot / V / Q^{*}$ & $-\cdot|r| \Lambda^{* m}$ & عوامل محيطى \\
\hline & & 1 & $\cdot /$ FAY"* & $\cdot|\Delta F|^{*}$ &.$/ l F r$ & $\cdot \mid \Delta T Y^{*}$ & $\cdot \mid \Delta \Delta 9^{* a t}$ & 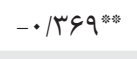 & روابط \\
\hline & 1 & $\cdot / V \vee 9^{* *}$ & . KYr"w & $\cdot / 49 . * *$ & $\cdot / 1 \Delta \Delta$ & $\cdot|K T|^{* * *}$ & $\cdot / K T D^{m * n}$ & $-\cdot / 199^{\circ}$ & 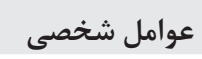 \\
\hline$(\cdot / A F)$ & $\cdot \mid \Delta \vee \Lambda^{* * *}$ & $\cdot \mid \Delta r \gamma^{* * *}$ & $.1949^{*}$ & - /AFY" & $\cdot / V \Delta Y^{* * *}$ &.$|9| 1$ * & $\cdot / V \backslash \Lambda^{*}$ & $-\cdot 109 \cdot \cdots$ & استرس شغلى \\
\hline
\end{tabular}

Correlation is significant at the 0.05 level (-2tailed)

"Correlation is significant at the 0.01 level (-2tailed). 


\begin{tabular}{|c|c|c|}
\hline \multirow[b]{2}{*}{ تاييد/رد ارتباط } & \multicolumn{2}{|r|}{ جدول r: ضرائب استاندارد و معنا دارى مرتبط با مدلهاى اندازهيرى } \\
\hline & اعداد معنادارى & 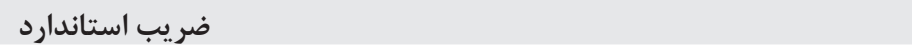 \\
\hline & & فرايند استرس شغلى \\
\hline تاييد & $r / \mathcal{A} \wedge$ & ماهيت كار استرس شغلى \\
\hline تاييد & G/rr & زمان كار ـ استرس شغلى \\
\hline تاييد & $r / r V$ & سياست سازمان ـ استرس شغلى \\
\hline تاييد & $\Lambda / r \Lambda$ & جايغاه سازمانى ؟ استرس شغلى \\
\hline تاييد & $1 / 19$ & عوامل محيطى ـ استرس شغلى \\
\hline تاييد & $9 / T V$ & روابط ـ استرس شغلى \\
\hline تاييد & $\Delta / \& q$ & عوامل شخصى ـ استرس شغلى \\
\hline \multicolumn{3}{|c|}{$\mathrm{RMSEA}=0.085$ ، GFI $=0.93$ ، AGFI $=0.90 ، \mathrm{NFI}=0.90, \mathrm{NNFI}=0.92$} \\
\hline \multicolumn{3}{|c|}{ 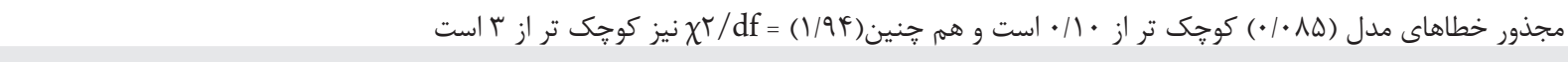 } \\
\hline & & رفتار شهروندى سازمانى \\
\hline تاييد & $\Delta / V$ & نوع دوستى ؟ رفتار شهروندى سازمانى \\
\hline تاييد & r/AF & وظيفه شناسى ؟ رفتار شهروندى سازمانى \\
\hline تاييد & $\Delta / r \Delta$ & جوانمردى ؟ رفتار شهروندى سازمانى \\
\hline تاييد & r/T & تواضع ؟ رفتار شهروندى سازمانى \\
\hline عدم تاييد & $1 / 8 \Lambda$ & عفت ؟ رفتار شهروندى سازمانى \\
\hline \multicolumn{3}{|c|}{$\mathrm{RMSEA}=0.077$ ،GFI $=0.97$ ، AGFI $=0.90 ، \mathrm{NFI}=0.92, \mathrm{NNFI}=0.91$} \\
\hline & 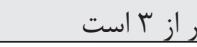 & 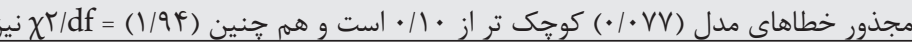 \\
\hline
\end{tabular}

\begin{tabular}{|c|c|c|c|}
\hline & & & جدول r: شاخصهاى برازش مدل ساختارى \\
\hline تأييد/رد ارتباط & اعداد معنادارى & ضريب استاندارد & سترس ـ رفتار شهروندى سازمانى \\
\hline تأييد & $-F / \cdot V$ & -.199 & ماهيت كار — ـرفتار شهروندى سازمانى \\
\hline تأييد & $-k / V \varepsilon$ & $-\cdot / r \Delta$ & زمان كار — † رفتار شهروندى سازمانى \\
\hline 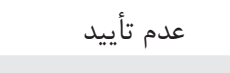 & $-1 / 9 \mathrm{~V}$ & $-\cdot 1 \cdot \mathrm{V}$ & سياست سازمان ٪ رفتار شهروندى سازمانى \\
\hline تأييد & $-r / \Lambda F$ & $-\cdot 1 \cdot 1$ & جايگاه سازمانى ـرفتار شهروندى سازمانى \\
\hline 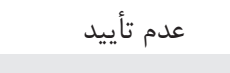 & $-1 / 1$ & $-\cdot 1 \cdot 0$ & عوامل محيطى ـ رفتار شهروندى سازمانى \\
\hline تأييد & $-4 / \pi q$ & -.190 & روابط سازمانى ـ ـرفتار شهروندى سازمانى \\
\hline عدم تأييد & $-1 / 19$ & $-\cdot / 4$ & عوامل شخصى — رفتار شهروندى سازمانى \\
\hline \multicolumn{4}{|c|}{ RMSEA $=0.064 ،$ GFI $=0.94$ ، AGFI $=0.91$ ، NFI $=0.95, \mathrm{NNFI}=0.97$} \\
\hline \multicolumn{4}{|c|}{ 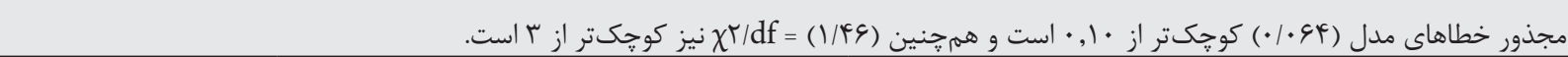 } \\
\hline
\end{tabular}

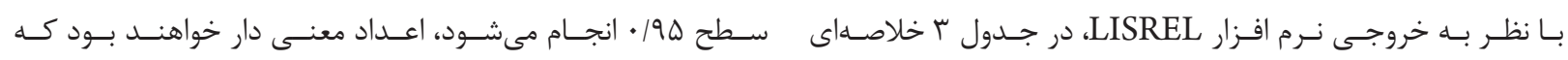

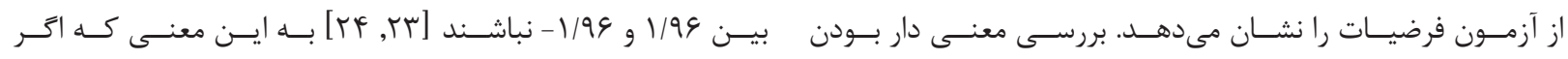

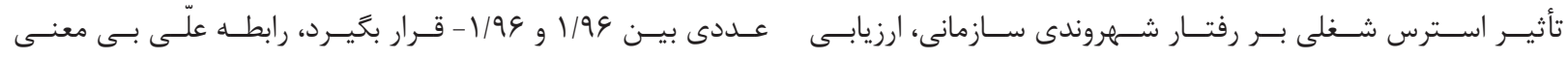

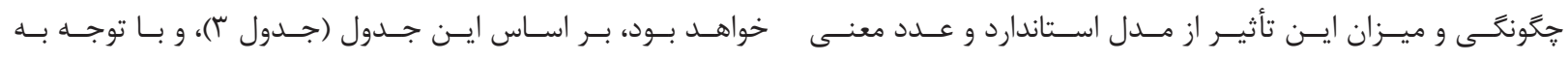

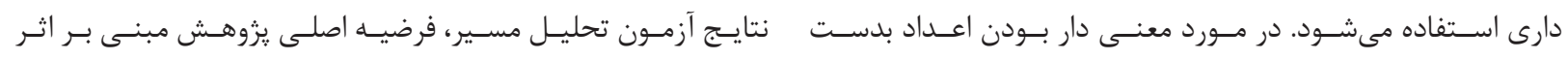

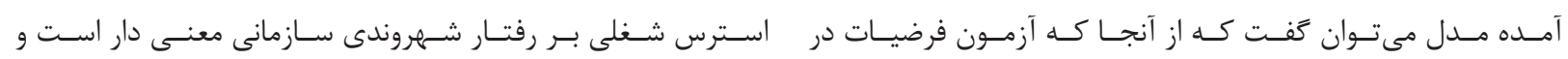




\section{نتيجه كيرى}

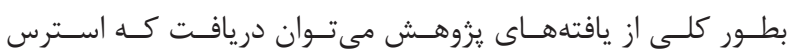

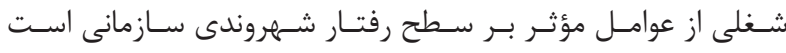

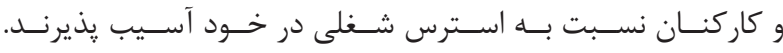

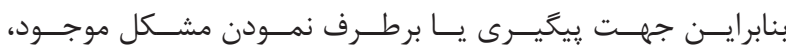

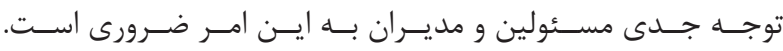

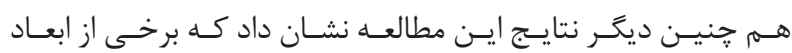

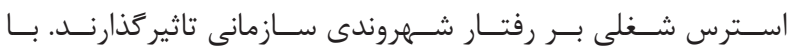

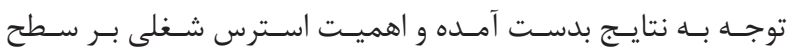

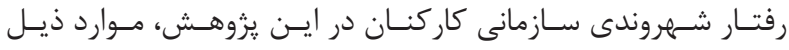

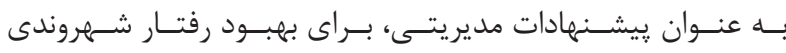

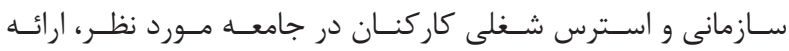

مى شـون:

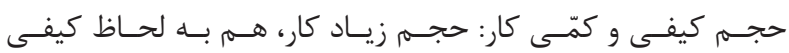

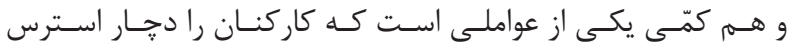

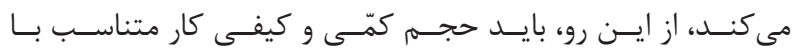

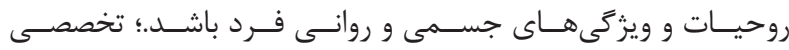

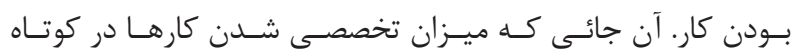

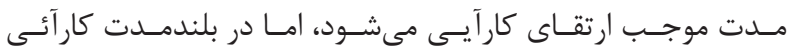

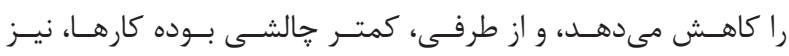

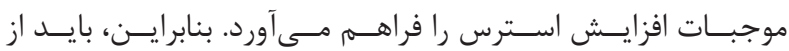

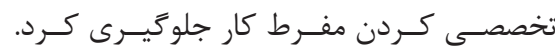

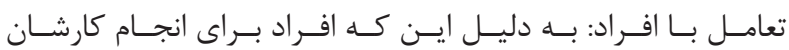

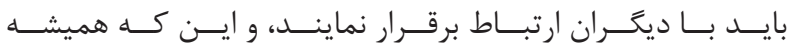

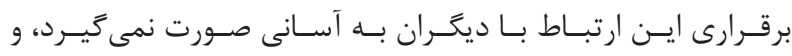

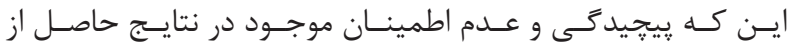

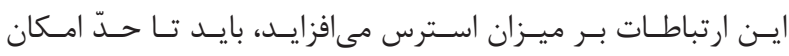

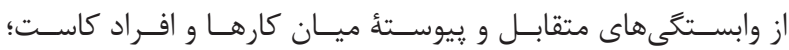

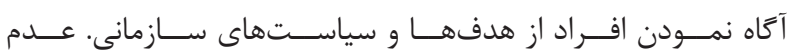

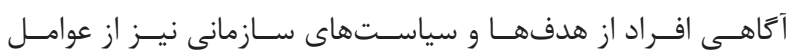

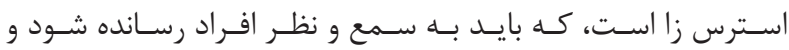

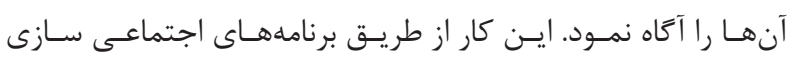

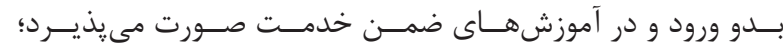

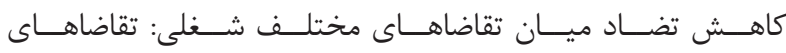

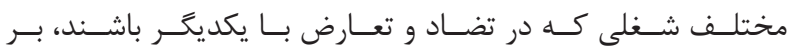

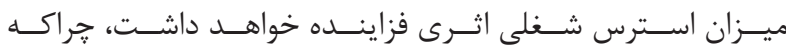

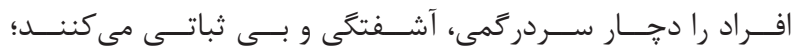

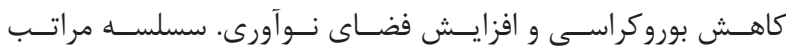

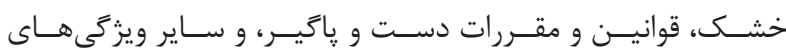

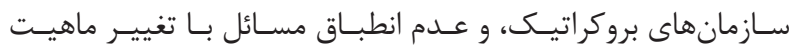

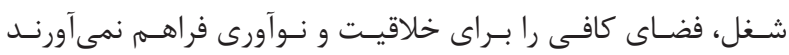

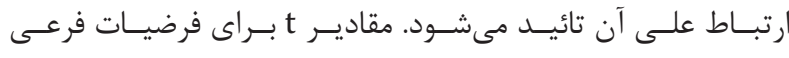

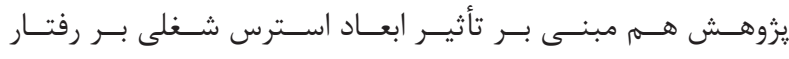

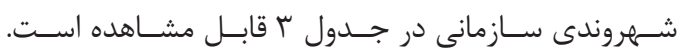

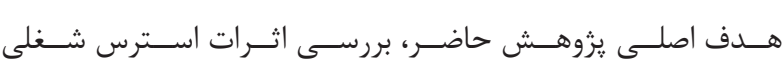

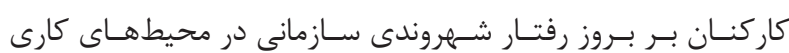

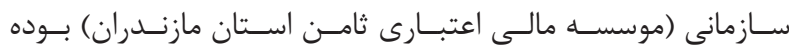

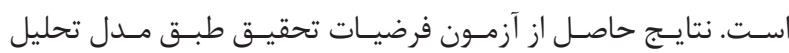

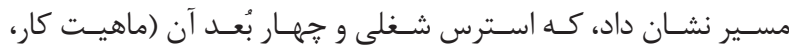

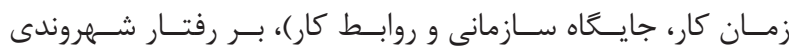

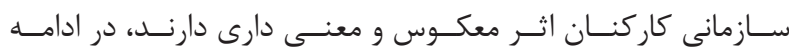

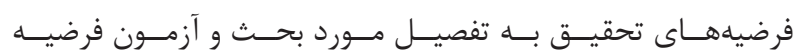

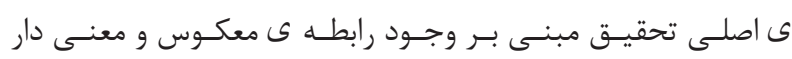

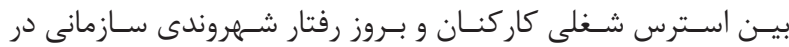

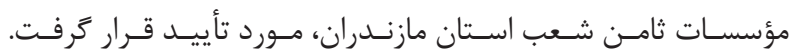

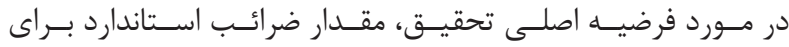

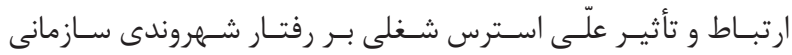

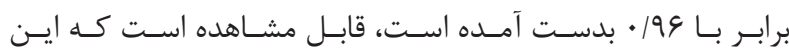

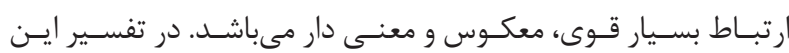

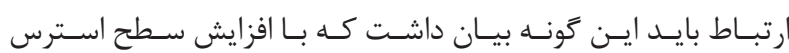

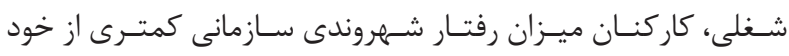

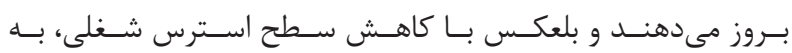

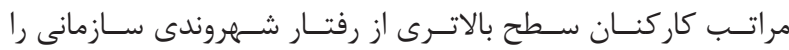

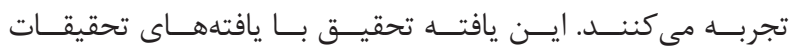

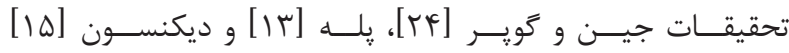

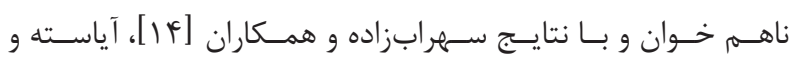

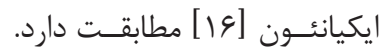

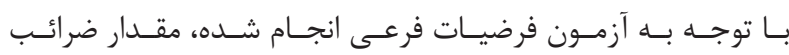

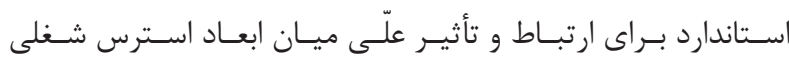

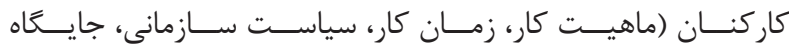

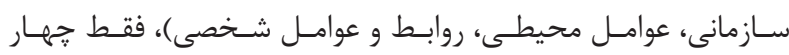

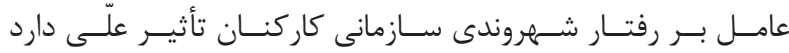

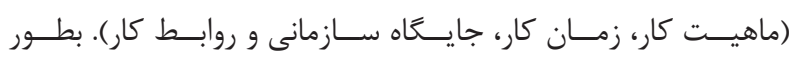

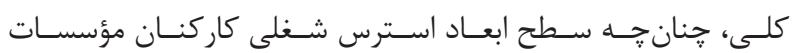

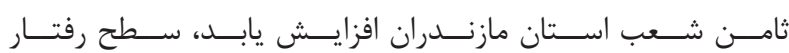

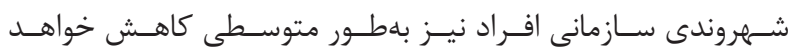

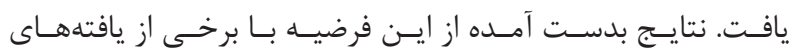

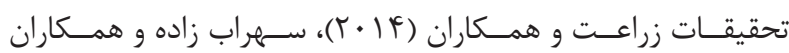

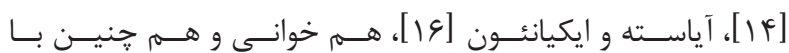

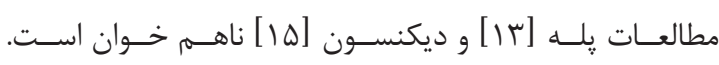




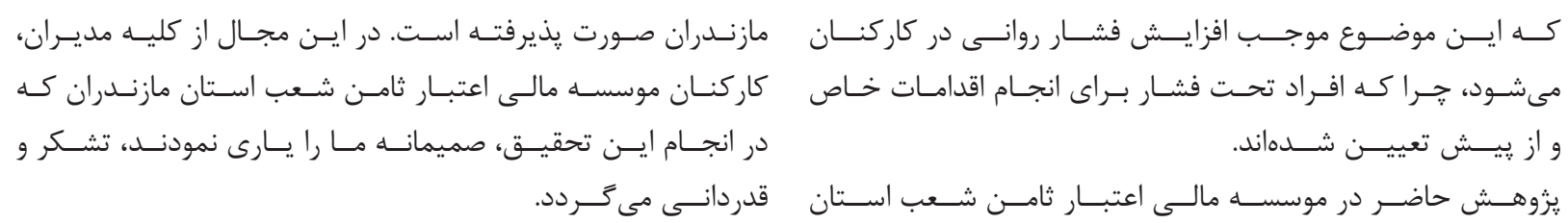

\section{REFERENCES}

1. DiPaola MF, Hoy WK. Organizational citizenship of faculty and achievement of high school students. High Sch J. 2005;88(3):3544. DOI: $10.1353 / \mathrm{hsj} .2005 .0002$

2. Markóczy L, Xin K. University of California [Internet]. California: university of California. 2004.

3. Katz D, Kahn R. The social psychology of organizations. New York: Wiley 1966.

4. Blakely GL, Andrews MC, Moorman RH. The moderating effects of equity sensitivity on the relationship between organizational justice and organizational citizenship behaviors. J Bus Psychol. 2005;20(2):259-73. DOI: 10.1007/s10869-005-8263-3

5. Podsakoff PM, MacKenzie SB, Paine JB, Bachrach DG. Organizational citizenship behaviors: A critical review of the theoretical and empirical literature and suggestions for future research. J Manage. 2000;26(3):513-63. DOI: 10.1177/014920630002600307

6. Levine EL. Emotion and power (as social influence): Their impact on organizational citizenship and counterproductive individual and organizational behavior. Hum Resour Manage Rev. 2010;20(1):417.

7. Wang J, Wong C. Understanding organizational citizenship behavior from a cultural perspective: An empirical study within the context of hotels in Mainland China. Int J Hosp Manag. 2011;30(4):84554.

8. Hamdi K, Moradi S. [The relationship between organizational citizenship behavior and organizational commitment]Persian. Beyond Manage J. 2009;4:33-50.

9. Hamdi K, Moradi S. [Job satisfaction and job stress among staff of health centers]Persian. Beyond Manage J. 2011;4:33-50.

10. Daniali SS, Amidimazaheri M, Mostafavi F, Hassanzadeh A, Tavassoli E. [Job satisfaction and job stress among staff of health center] [Persian. J Health Sys Res 2014;10(1):1-10.

11. Barzideh M, Choobineh A, Tabatabaei SH. [Job stress dimensions and their relationship to job change intention among nurses]persian. Journal of Ergon. 2013;1(1):33-42.

12. Safari S, Habibi E, H. D, Mahaki B, Hassanzade A. [Job stress, employee education and ability to work in a refinery]Persian. J Occup Med Specialist. 2013;5(3):1-10.
13. Pallie P. Stressful work, citizenship behaviour and intention to leave the organization in a high turnover environment: examining the mediating role of job satisfaction. J Manage Res. 2011;3(1):1-14

14. Ayatse FA, Ikyanyon DN. Organizational communication, job stress and citizenship behaviour of IT employees in Nigerian Universities. J Bus Adm Res. 2012;1(1):99. DOI: 10.5430/jbar.v1n1p99 citizenship behavior. The University of Tennessee at Chattanooga: Departmental Honors Thesis; 2009.

16. Sohrabizade S, Bastani P, Ravangard R. [Factors influencing organimedical sciences Shiraz]Persian. J hospital 2010;1(2):82-75.

17. Niehoff B, Moorman R. Justice as a mediator of the relationship between methods of monitoring and organizational citizenship behavior. Acad Manage J. 1993;36(3):527-56. DOI: 10.2307/256591

18. NG S, Skitmore M, Leung R. Manageability of stress among construction project participants. Engin Constr Archit Manag. 2005;12(9):264-82. DOI: 10.1108/09699980510600125

19. Bollen KA. Structural equations with latent variables. New York Wiley; 1981.

20. Gerbing DW, Anderson JC. An updated paradigm for scale development incorporating unidimensionality and its assessment. J Mark Res. 1988;18:186-92. DOI: $10.2307 / 3150980$

21. Gerbing DW, Anderson JC. An updated paradigm for scale development incorporating unidimensionality and its assessment. J Mark Res. 1988;25(2):186-92. DOI: $10.2307 / 3172650$

22. Steenkamp J, Van Trijp H. The use of LISREL in validating marketing constructs. Int J Res Mark 1991;8(4):283-99. DOI: $\underline{10.1016 / 0167-8116(91) 90027-5}$

23. $\mathrm{Hu} \mathrm{L}, \mathrm{Bentler}$ PM. Cutoff criteria for fit indexes in covariance structure analysis: Conventional criteria versus new alternatives. Struct Equ Model Multidiscip J. 1999;6(1):1-55. DOI: $10.1080 / 10705519909540118$

24. Jain AK, Cooper CL. Stress and organisational citizenship behaviours in Indian business process outsourcing organisations. IIMB Manage Rev. 2012;24(3):155-63. DOI: $10.1016 / \mathrm{j}$. iimb.2012.06.004
15. Dickinson L. An examination of the factors affecting organizational zational citizenship behavior of employees ministerial university of 


\title{
The Effects of Job Stress on the Emersion of Employees' Organizational Citizenship Behavior in a Finance and Credit Institute in Mazandaran Province
}

\author{
Hossein Samadi Miarkolaei ${ }^{1,}{ }^{*}$, Hamzeh Samadi Miarkolaei ${ }^{2}$ \\ ${ }^{1}$ PhD Student of Public Administration, Young Researchers and Elite Club, \\ Qaemshahr Branch, Islamic Azad University, Qaemshahr, Iran \\ ${ }^{2}$ PhD of Public Administration, Young Researchers and Elite Club, Qaemshahr \\ Branch, Islamic Azad University, Qaemshahr, Iran \\ * Corresponding author: Hossein Samadi Miarkolaei, PhD Student of Public Ad- \\ ministration, Young Researchers and Elite Club, Qaemshahr Branch, Islamic Azad \\ University, Qaemshahr, Iran.E-mail:hossein_samadi_m@yahoo.com
}

DOI: $10.21859 /$ joe- 040430

Received: 13.03 .2016

Accepted: 26.07.2016

\section{Keywords:}

Job Stress

Organizational Citizenship Behavior

Work Environment

Employees

How to Cite this Article:

Samadi Miarkolaei H, Samadi Miarkolaei H. The Effects of Job Stress on the Emersion of Employees' Organizational Citizenship Behavior in a Finance and Credit Institute in Mazandaran Province. J Ergo. 2016;4(2):52-59. DOI: 10.21859/joe-0402430

(C) 2016 Hamedan University of Medical Sciences.

\section{Abstract}

Introduction: Job stress is identified as imbalance between job needs and individual's abilities and demands. Job stress has somatic, psychological and behavioral traumas, The main purpose of the present research was to investigate the effects of employees' job stress level on the emersion of organizational behavior amongst Mazandaran's SAMEN Finance and Credit Institute's subdivision employees.

Methods: In this correlation-descriptive study, 147 employees from SAMEN subdivisions of Mazandaran province were selected based on the census sampling method. Data were collected by using the job stress and organizational citizenship behavior standard questionnaire. Data were analyzed by the SPSS and LISREL software, and using descriptive statistics, Pearson's correlation and Standard Error of the Mean (SEM).

Results: In the current study, there was a significant and inverse relationship between job stress and organizational citizenship behavior $(R=-0.590$, and $P=0.001)$. The results of structural equation modeling and path analysis showed that job stress and most of its components were good predictors of organizational citizenship behavior.

Conclusions: Recent research has demonstrated that job stress may increase employeesrelated problems. There is a relationship between job stress and organizational citizenship behavior, and attention to job stress level as a predictor of organizational citizenship behavior in employees is an essential issue. 